\title{
Cyclooxygenase-2 expression in squamous cell carcinoma of the oral cavity and pharynx: Association to p53 and clinical outcome
}

\author{
TIMO ATULA ${ }^{1}$, JOHAN HEDSTRÖM ${ }^{1,2}$, ARI RISTIMÄKI ${ }^{4,5}$, PATRIK FINNE $^{2}$, \\ ILMO LEIVO $^{4}$, MARI MARKKANEN-LEPPÄNEN ${ }^{1}$ and CAJ HAGLUND ${ }^{3,4}$
}

\author{
${ }^{1}$ Department of Otorhinolaryngology - Head and Neck Surgery, Helsinki University Central Hospital, P.O.Box 220; \\ ${ }^{2}$ Department of Clinical Chemistry, Biomedicum Helsinki, P.O. Box 700; ${ }^{3}$ Department of Surgery, \\ Helsinki University Central Hospital, P.O.Box 340, FIN-00029 HUS; ${ }^{4}$ Department of Pathology, \\ Helsinki University Central Hospital; ${ }^{5}$ Cancer Biology Research Program, \\ Biomedicum Helsinki, University of Helsinki, Helsinki, Finland
}

Received March 2, 2006; Accepted May 22, 2006

\begin{abstract}
Cyclooxygenase-2 (COX-2) expression is upregulated in transformed cells and in malignant tissues, including tumours of the head and neck, and it has prognostic significance in many types of cancer. COX-2 expression is suppressed by the wild-type but not by the mutant tumour suppressor gene TP53. The purpose of this study was to investigate the association between the expression of COX-2 and the clinical outcome in patients with oral and pharyngeal squamous cell carcinoma (SCC), and to examine its relationship to p53. Immunohistochemistry showed an elevated COX-2 expression in $88 \%$ ( $n=57$; strong 38 , weak 19) of the 65 tumour samples. The staining intensity was not associated with patient or tumour characteristics, nor with the immuhistochemical expression of p53. Kaplan-Meier analysis showed no significant correlation between $\mathrm{COX}-2$ expression and recurrence-free or overall survival, but a strong p53 expression was associated with a poor recurrence-free $(\mathrm{p}=0.001, \log$-rank) and overall survival $(\mathrm{p}=0.003)$. We conclude that, unlike strong p53 expression, COX-2 expression does not have prognostic significance in advanced oral and pharyngeal SCCs.
\end{abstract}

\section{Introduction}

Despite the development in treatment strategies, patients with advanced oral and pharyngeal squamous cell carcinoma (SCC) have a poor prognosis. The overall 5 -year survival rate in stage III and IV disease is less than $40 \%$ (1). The TNM

Correspondence to: Dr Timo Atula, Department of Otorhinolaryngology, Head and Neck Surgery, P.O. Box 220, FIN-00029 HUS, Helsinki, Finland

E-mail: timo.atula@hus.fi

Key words: cyclooxygenase-2, head and neck cancer, prognosis, malignancy, survival classification does not reliably predict the clinical outcome in individual patients, and recent research has focused on targeted therapies (1-4). Therefore, there is a need to further investigate molecular markers associated with the biological behaviour of the tumour.

Epidemiological studies suggest that the use of nonsteroid anti-inflammatory drugs (NSAIDs) is associated with a reduced risk of cancer especially in the gastrointestinal tract (5). The best known target of NSAIDs is cyclooxygenase $(\mathrm{COX})$, the rate-limiting enzyme in the conversion of arachidonic acid to prostanoids $(6,7)$. Of the two known isoforms of COX, the expression of COX-2 is low or not detectable in most healthy tissues, but can be highly induced in response to cell activation by hormones, proinflammatory cytokines, growth factors, and tumour promoters $(6,7)$. Through different mediators, COX-2 expression promotes angiogenesis, invasiveness, cell proliferation, immunosuppression and inhibits apoptosis $(6,8)$. These many important processes in carcinogenesis make COX-2 an attractive therapeutic target $(4,6-9)$.

Elevated COX-2 expression has been described in a wide variety of human premalignant and malignant conditions and in experimental animal models of carcinogenesis $(6,7,10)$. COX-2 upregulation has been shown in head and neck squamous cell carcinomas (HNSCC) at both mRNA and protein levels $(11,12)$, and it contributes to the growth and progression of HNSCC through multiple pathways $(11,13-$ 15).

Tumour suppressor gene TP53 is commonly mutated in human malignancies, which leads to the accumulation of a mutant non-functional p53 protein $(5,14,16)$. TP53 mutations and altered $\mathrm{p} 53$ expression have often been associated with poor prognosis in HNSCC patients (2,16-20). p53 status is also associated with COX-2 expression (14,21-23), and the wild-type but not mutant $\mathrm{p} 53$ has been shown to suppress COX-2 transcription (21), and moreover, overexpression of COX-2 has been detected in HNSCCs containing mutant TP53 (14). 
As in various other cancer forms, the role of COX-2 as a possible target for inhibiting the growth of HNSCCs has been examined $(7,9,24,25)$. Experimental studies show that COX-2 inhibition can suppress the growth of human squamous carcinoma cells $(9,12,24,26)$, further supporting the role of COX-2 in HNSCC.

The prognostic significance of COX-2 has been shown especially in certain types of adenocarcinoma (10). Its prognostic significance in SCC is less investigated. In esophageal SCCs, it does not seem to have prognostic value (27). The prognostic significance of COX-2 in HNSCC has been examined by three groups reporting controversial results $(13,15,28)$. For this reason, we investigated the association between the expression of COX-2 and the clinical outcome in patients with SCC of the oral cavity and pharynx, and in addition, the association between p53 and COX-2 expression.

\section{Patients and methods}

Patients and tumours. Formalin-fixed and paraffin-embedded tissue specimens from 65 patients with previously untreated invasive SCC of the oral cavity and pharynx were obtained from the files of the Department of Pathology, Helsinki University Central Hospital. The samples were originally drawn from a consecutive series of patients undergoing primary radical surgery including microvascular free-flap reconstruction at Helsinki University Central Hospital between 1989 and 1998. All samples were re-evaluated by the pathologist experienced in head and neck pathology (I.L.) to confirm the diagnosis. The data collected for each patient included age, sex, primary tumour site, tumour size, cervical nodal status, overall TNM classification and tumour stage, histological grade, treatment, tumour recurrence, and cause of death. The mean age at the time of diagnosis was 58 years (range 31-80). Twenty-one of the patients were women and 44 men. Of the 65 patients, $32(49 \%)$ had a carcinoma in the oral cavity, $19(29 \%)$ in the oropharynx, and $14(22 \%)$ in the hypopharynx. According to the UICC (Unite international contre le cancer) TNM classification, none had stage I, 10 (15\%) had stage II, 23 (35\%) had stage III, and 32 (49\%) had stage IV cancer. Thirty-eight patients $(58 \%)$ had metastases in the neck and none had distant metastases at the time of diagnosis. Four patients $(6 \%)$ received radiotherapy preoperatively, 55 patients $(85 \%)$ postoperatively, whereas 6 patients $(9 \%)$ underwent surgical treatment only. The median follow-up time was 1.49 years (range 0.14-9.9) for all the patients, and 2.1 years (range 0.64-9.9) for those who survived until the end of the follow-up.

Immunohistochemistry. The specimens were sectioned $(4 \mu \mathrm{m})$, deparaffinized, and microwaved for $4 \times 5 \mathrm{~min}$ at $700 \mathrm{~W}$ in $0.01 \mathrm{~mol} / \mathrm{l} \mathrm{Na}$-citrate buffer $(\mathrm{pH} 6.0)$ for antigen retrieval. The slides were immersed in $0.6 \%$ hydrogen peroxide in methanol for $30 \mathrm{~min}$ to block endogenous peroxidase activity and then in blocking solution [1.5:100 normal horse serum in phosphate-buffered saline (PBS)] for $15 \mathrm{~min}$ to block non-specific binding sites. Immunostaining was performed with a COX-2-specific anti-human monoclonal antibody (160112; Cayman Chemical Co., Ann Arbor, MI) in a dilution of $1: 200(2.5 \mu \mathrm{g} / \mathrm{ml})$ in PBS containing $0.1 \%$ sodium azide and $0.5 \%$ bovine serum albumin at room temperature overnight. Then the sections were treated with biotinylated horse anti-mouse immunoglobulin (1:200; Vector Laboratories Inc., Burlingame, CA) and avidin-biotin peroxidase complex (Vectastain ABComplex, Vector Laboratories). The peroxidase staining was visualized with 3amino-9-ethylcarbazole (Sigma Chemical Co., St. Louis, MO). Counterstaining was performed with Mayer's hematoxylin. For the control, the primary antibody was preadsorbed with human COX-2 control peptide $(1-10 \mu \mathrm{g} / \mathrm{ml}$, Cayman Chemical) for $1 \mathrm{~h}$ at room temperature prior to the staining procedure. $\alpha$-smooth muscle cell actin peptide (50 $\mu \mathrm{g} / \mathrm{ml}$; Dako, Glostrup, Denmark) was used as a non-COX-2 peptide. The intensity of staining was scored into four groups; 0 , no staining; 1 , weak diffuse cytoplasmic staining (may contain stronger intensity in $<10 \%$ of the cancer cells); 2 , moderate/strong granular cytoplasmic staining in $>10 \%$ of the cancer cells; 3 ; intense granular cytoplasmic staining in $>50 \%$ of the cancer cells. The slides were scored by two pathologists (I.L. and A.R.) unaware of the clinical data.

The principle of immunohistochemistry for p53 (clone DO7, Dako), which recognizes both the mutant and wildtypes of p53 was similar to that described for COX-2. The primary antibody was diluted 1:300. For antigen retrieval of $\mathrm{p} 53$, the sections were processed in a $700 \mathrm{~W}$ microwave oven for $4 \times 5 \mathrm{~min}$ in a $0.3 \%$ citrate buffer ( $\mathrm{pH} 6.0$ ). Formalin-fixed paraffin-embedded sections of a known breast cancer specimen positive for the antigen served as the positive control in every staining batch. Sections treated with PBS instead of the primary antibody served as the negative controls. Staining for p53 was considered weak when $<20 \%$ and strong when $>20 \%$ of cancer cell nuclei showed staining (29).

Statistical analysis. The relationship between the staining intensity and pattern of COX-2 and p53, sex, age ( $>60$ years), tumour site, grade, size (T-status), metastases (N-status), and stage were assessed with the Chi-square test. Fisher's exact test was used when necessary because of low expected values. Overall survival time was defined as the interval between primary surgical treatment and death or the end of follow-up (event: death, censored at the end of follow-up). Correspondingly, the end-point for recurrence-free survival time was the detection of recurrent disease or the end of follow-up (event: recurrence of disease, censored at the end of follow-up). Probabilities of overall and recurrence-free survival were calculated according to the Kaplan-Meier method. Differences in survival probability between the groups were assessed using the log-rank test. The Cox proportional hazards regression was used to estimate relative risks adjusted for other prognostic factors. All p-values were two-tailed, and $\mathrm{p}<0.05$ was considered statistically significant.

\section{Results}

Expression of COX-2 and p53. Staining for COX-2 was detected in $88 \%$ (57 of 65) of the SCC samples (Fig. 1). The staining was negative (scored 0 ) in 11\% (7/65), weak (score 1) in $58 \%(38 / 65)$, strong (score 2$)$ in $28 \%(18 / 65)$, and intense 

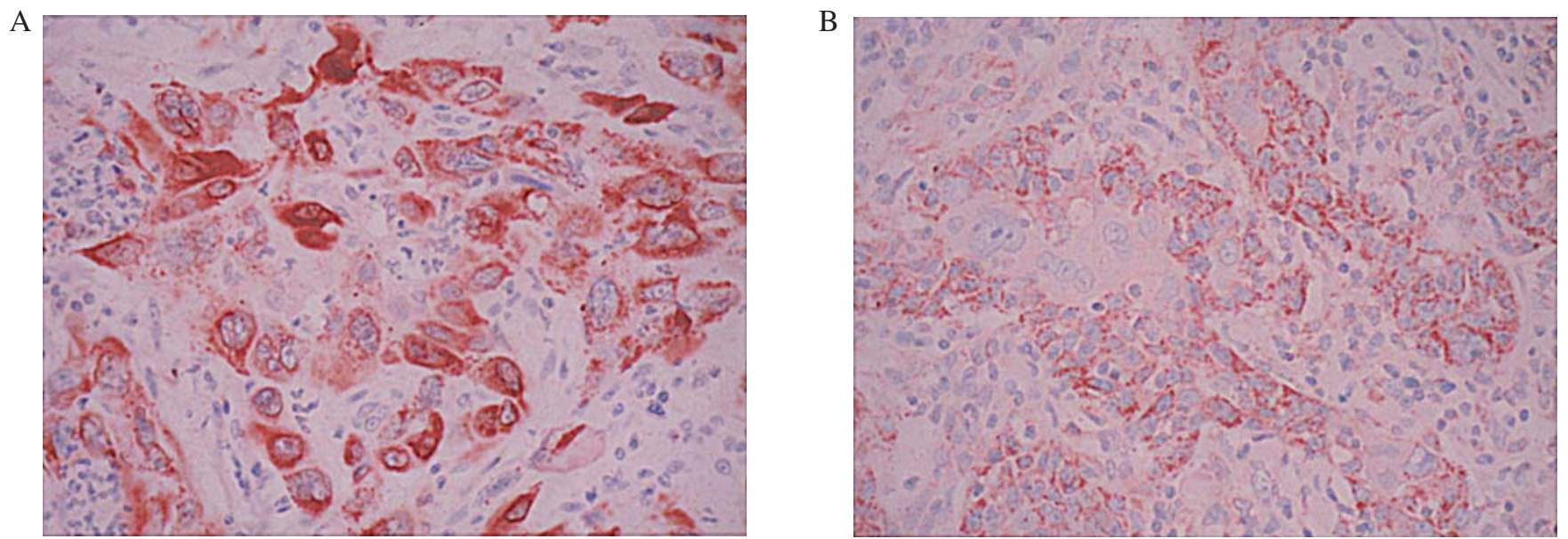

Figure 1. Immunohistochemistry for cyclooxygenase-2 (COX-2) in oral squamous cell carcinoma. (A) Strong cytoplasmic immunostaining is visible in moderately differentiated carcinoma cells. (B) Weak cytoplasmic immunostaining is seen in well-differentiated squamous cell carcinoma

(score 3$)$ in $1.5 \%(1 / 65)$ of the samples. For statistical analysis, COX-2 scores 0 and 1 (no staining or weak staining) and likewise scores 2 and 3 (strong and intense staining) were combined. The staining intensity for $\mathrm{COX}-2$ showed no significant correlation with patients' sex or age, tumour site, grade, size (T-status), metastases ( $\mathrm{N}$ status) or stage.

p53 expression showed no staining in 30\% (19/63), it was weak in $29 \%(18 / 63)$ and strong in $41 \%(26 / 63)$ of the samples. For statistical analysis of p53, samples with no staining or weak staining were combined and compared with those with strong staining. p53 expression did not correlate with patients' sex or age, tumour site, grade, size (T-status), metastases ( $\mathrm{N}$ status) or stage, and nor with $\mathrm{COX}-2$ staining $(\mathrm{p}=0.64)$.

Association with clinical outcome. High tumour stage correlated with poor overall survival $(\mathrm{p}=0.044)$ while the $\mathrm{T}$ status alone did not predict survival. Nodal status of N2 or N3 was associated with a shorter overall survival than the nodal status of $\mathrm{N} 1$ or NO ( $\mathrm{p}=0.006)$. Tumour grade showed a weak trend with overall survival $(p=0.063)$. Tumours in the oropharynx were associated with more favorable recurrencefree $(\mathrm{P}=0.018)$ and overall survivals $(\mathrm{p}=0.043)$ than oral or hypopharyngeal tumours, but until a follow-up time of two years these showed only small differences in survival rates.

For all the patients, the probability of surviving two years was 0.53 (95\% CI $0.40-0.65)$, while the probability of recurrence-free survival was 0.48 (95\% CI 0.35-0.62). COX-2 staining intensity did not correlate significantly with recurrence-free $(\mathrm{p}=0.11, \log$-rank test $)$ or overall $(\mathrm{p}=0.75)$ survival (Fig. 2). The 2-year recurrence-free survival rates were 0.64 for the strong staining group and 0.43 for patients with weak or no staining. The corresponding rates for overall survival were 0.56 and 0.52 .

The staining of p53 correlated with recurrence-free survival ( $p=0.001$, log-rank test) and overall survival $(p=0.003)$ (Fig. 3). When combining COX-2 staining intensity and p53 in a Cox regression analysis, p53 remained significant (relative risk $2.4,95 \%$ CI $1.3-4.4, \mathrm{p}=0.004)$, while COX-2 (strong vs weak or no staining) did not $(\mathrm{p}=0.996)$.

\section{Discussion}

We examined the association between COX-2 expression and patient outcome in oral and pharyngeal SCC, but found no correlation with recurrence-free or overall survival. In contrast to our results, elevated COX-2 expression is associated with unfavourable outcome in various cancers, such as gastrointestinal, breast and ovarian cancer $(10,22)$. Three previous reports dealing with the prognostic significance of COX-2 in HNSCC show contradictory results $(13,15,28)$. In a study by Ranelletti et al (28), including an immunohistochemical analysis of 61 laryngeal SCCs, stronger COX-2 staining was associated with a more favourable overall survival and reduced risk of tumour recurrence. In another study by Gallo et al (13), immunohistochemical analysis of 52 SCCs of the oral cavity, oropharynx, and larynx showed that strong COX-2 staining was associated with a reduced disease-free and overall survival. In a recent study of 70 patients including oral, laryngeal and a large number of lip carcinomas, COX-2 expression alone did not correlate with survival, but it was a sign for poor prognosis when combined with VEGF-C (15).

Differences in results between the studies may partly be explained by different kinds of patient materials. In addition to different distributions of tumour sites, our material consisted of more advanced tumours. In other studies, the proportion of stage I or II diseases varied from $23 \%$ (13) to $66 \%$ (15), while we had no stage I tumours and only $15 \%$ stage II tumours. There were also methodological differences between the studies. i) The antibodies were different. ii) A computer-based analysis of sample staining intensity was used in one study, and different cut-off values were tested in the survival analysis (28). In other studies, including ours, staining was analyzed by pathologists unaware of the clinical data. In our series, COX-2 was expressed in the majority $(88 \%)$ of SCC samples as detected by immunohistochemistry. This is in line with other studies showing COX-2 overexpression from 44 to $96 \%$ of HNSCCs $(15,30)$. COX-2 staining was localized to the cytoplasm of neoplastic cells, whereas the tumour stroma remained unstained. Normal squamous epithelium neighbouring the tumour showed weak 
A

Probability of overall survival

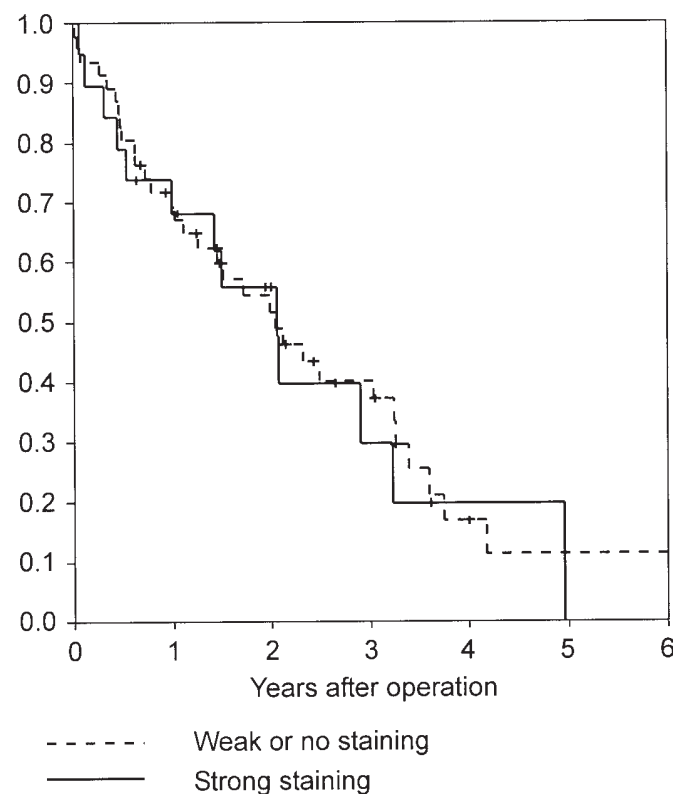

B

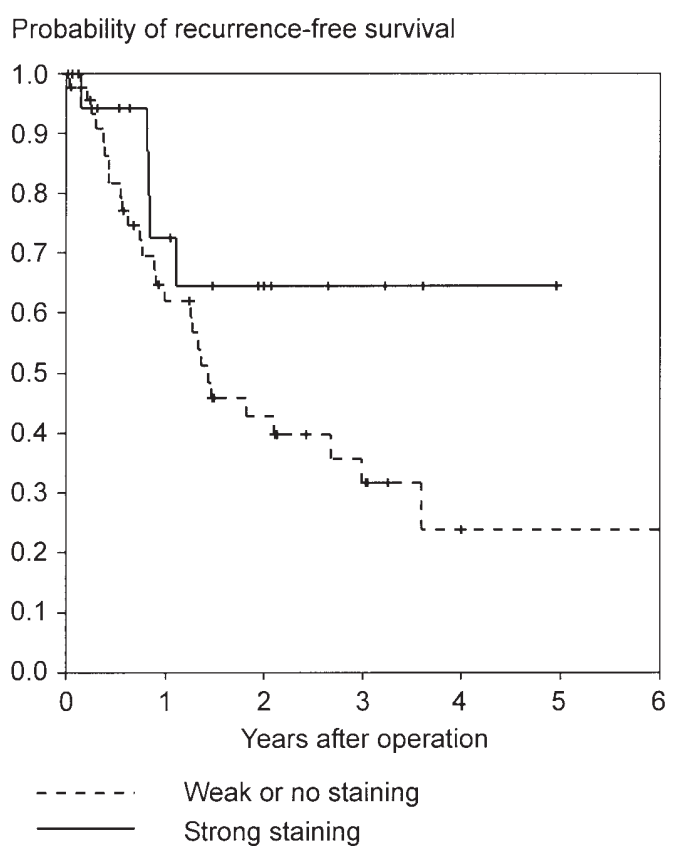

Figure 2. Staining intensity of COX-2 in tumour cells. (A) Kaplan-Meier curves for the corresponding overall survival (log-rank test, $\mathrm{p}=0.75$ ). (B) Kaplan-Meier curves for the corresponding recurrence-free survival (log-rank test, $\mathrm{p}=0.11$ ).

A

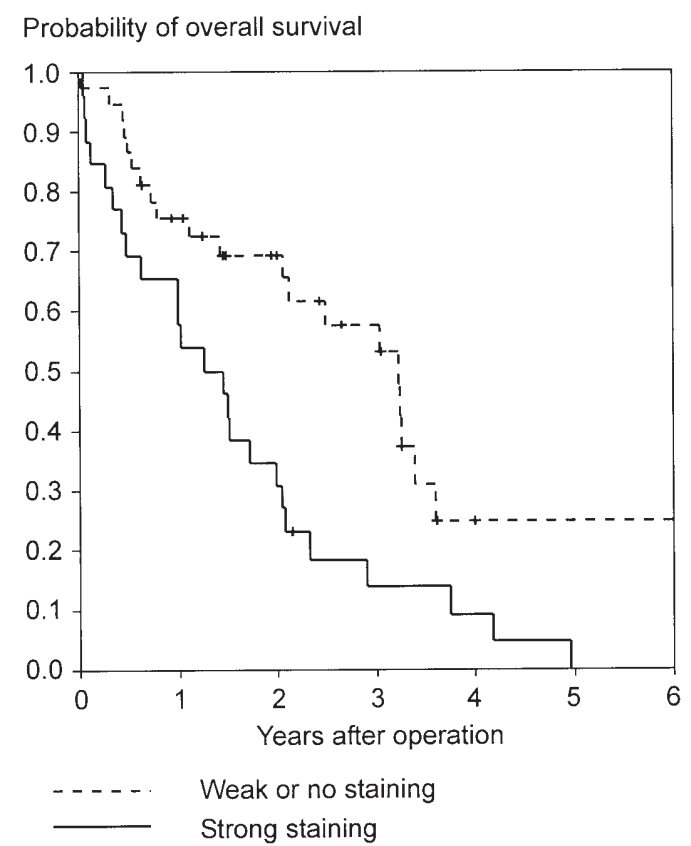

B

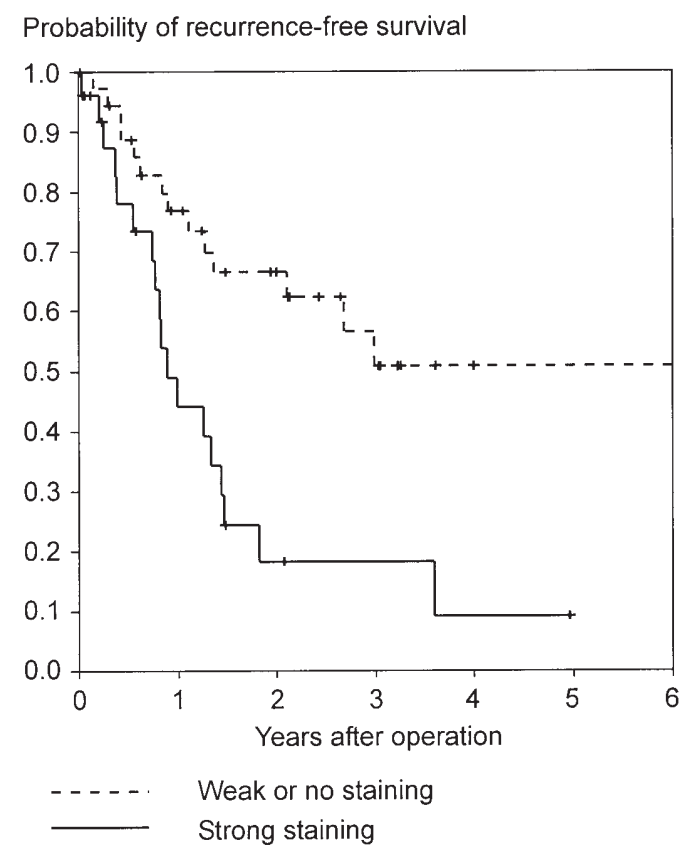

Figure 3. Staining intensity of p53 in tumour cells. (A) Kaplan-Meier curves for the corresponding overall survival (log-rank test, p=0.003). (B) Kaplan-Meier curves for the corresponding recurrence-free survival (log-rank test, $\mathrm{p}=0.001)$.

or no expression of COX-2. This is also in accordance with earlier studies of tumour specimens from $\operatorname{HNSCC}(12,13,30)$, nasopharyngeal carcinomas (31), as well as cancers from other anatomical and histological sites (10). However, the semiquantitive nature and limitations of immuhistochemistry may contribute to the discrepancies. Taken together, COX-2 expression does not have evident prognostic value in HNSCC, but obviously the number of patients included in studies on HNSCC is too limited to make definite conclusions. Furthermore, our study does not show whether COX-2 expression has prognostic significance at the early stage tumours. Reports on gastric and pulmonary 
adenocarcinomas indicate that COX-2 overexpression appears to be a marker for poor survival at early, but not at advanced stages $(10,32)$.

Lymph node metastasis is the most significant prognostic sign in HNSCCs, and not surprisingly, our material showed a correlation between metastases and poor survival. Association between COX-2 expression and the development of metastases in HNSCC has been reported previously $(15,30)$, but our study failed to support these findings.

Our study showed no significant association between COX-2 and p53 expression. To our knowledge, both p53 and COX-2 expressions have not been evaluated previously from the same HNSCC tumour specimens. Overexpression of p53 was detected in $70 \%$ of the tumours, which is consistent with other reports on HNSCC $(3,19,20)$. As carcinomas containing mutant TP53 have been demonstrated to overexpress COX-2 $(14,22,23)$, one could assume a correlation between the overexpression of $\mathrm{p} 53$ and COX-2. One explanation for the failure to demonstrate such a correlation may be the fact that $\mathrm{p} 53$ staining is not a direct marker for gene mutation $(17,33)$. In contrast to $\mathrm{COX}-2$, strong p53 immunostaining predicted a poor recurrence-free and overall survival. Previous studies on p53 expression and survival in HNSCC are inconclusive $(16,18-20)$, and the study settings variable, e.g. some reports focus on the expression of p53 in terms of response to radiation therapy $(16,19)$ or chemotherapy $(20)$. However, TP53 mutations are associated with poor survival (16-18).

The series of the samples for this study were collected from a period before chemoirradiation protocols (given either alone followed by salvage surgery when needed, or as a concomitant postoperative treatment) became part of the standard treatment strategy for these patients at our institution. Thus, the treatment of patients in our material is quite uniform. There is an ongoing search for molecular markers which could predict response to chemotherapy $(1,3,4)$. A recent study on esophageal SCCs showed an association of low COX-2 expression and poor prognosis in patients who had received neoadjuvant chemotherapy (cisplatin and etoposide) (27). This finding indicates that COX-2 may serve as a marker for favourable response to chemotherapy regimens.

To conclude, in our material of advanced oral and pharyngeal SCCs, the staining intensity of COX-2 did not correlate with prognosis. No correlation between COX-2 and p53 expression was found, but strong p53 expression was associated with tumour recurrence and poor overall survival. These findings do not rule out the possibility that COX-2 has a prognostic value at the earlier stages of the disease or in certain subgroups of patients, such as those with a favourable response to chemotherapy.

\section{Acknowledgements}

We thank Elina Laitinen and Sari Nieminen for excellent technical assistance. This study was supported by grants from Finska Läkaresällskapet, the Research Foundation of Helsinki University Central Hospital, Helsinki University Research Funds, the Research Foundation Liv och Hälsa, Sigrid Juselius Foundation, the Research Foundation K.A. Johansson and the Finnish Academy of Sciences. The study protocol was approved by the local ethics committee.

\section{References}

1. Lefebvre JL: Current clinical outcomes demand new treatment options for SCCHN. Ann Oncol 16 (suppl 6): vi7-vi12, 2005.

2. Salesiotis A and Cullen K: Molecular markers predictive of response and prognosis in the patient with advanced squamous cell carcinoma of the head and neck: evolution of a model beyond TNM staging. Curr Opin Oncol 12: 229-239, 2000.

3. Kim ES, Kies M and Herbst RS: Novel therapeutics for head and neck cancer. Curr Opin Oncol 14: 334-342, 2002.

4. Modjtahedi H: Molecular therapy of head and neck cancer. Cancer Metastasis Rev 24: 129-146, 2005.

5. Langman MJ, Cheng KK, Gilman EA and Lancashire RJ: Effect of anti-inflammatory drugs on overall risk of common cancer: case-control study in general practice research database. BMJ 320: 1642-1646, 2000.

6. Dannenberg AJ, Altorki NK, Boyle JO, et al: Cyclo-oxygenase2: a pharmacological target for the prevention of cancer. Lancet Oncol 2: 544-551, 2001.

7. Mohan S and Epstein JB: Carcinogenesis and cyclooxygenase: the potential role of COX-2 inhibition in upper aerodigestive tract cancer. Eur J Cancer B Oral Oncol 39: 537-546, 2003.

8. Lin DT, Subbaramaiah K, Shah JP, Dannenberg AJ and Boyle JO: Cyclooxygenase-2: a novel molecular target for the prevention and treatment of head and neck cancer. Head Neck 24: 792-799, 2002.

9. Zhang X, Chen Z, Choe MS, et al: Tumor growth inhibition by simultaneously blocking epidermal growth factor receptor and cyclooxygenase-2 in a xenograft model. Clin Cancer Res 11: 6261-6269, 2005.

10. Saukkonen K, Buskens CJ, Sivula A, et al: COX-2 in cancer. In: COX-2 inhibitors. Pairet $\mathrm{M}$ and van Ryn J (eds). Birkhäuser Verlag, Basel, pp227-243, 2004.

11. Chan G, Boyle JO, Yang EK, et al: Cyclooxygenase-2 expression is up-regulated in squamous cell carcinoma of the head and neck. Cancer Res 59: 991-994, 1999.

12. Mestre JR, Chan G, Zhang F, et al: Inhibition of cyclooxygenase-2 expression. An approach to preventing head and neck cancer. Ann N Y Acad Sci 889: 62-71, 1999.

13. Gallo O, Masini E, Bianchi B, Bruschini L, Paglierani M and Franchi A: Prognostic significance of cyclooxygenase-2 pathway and angiogenesis in head and neck squamous cell carcinoma. Hum Pathol 33: 708-714, 2002.

14. Gallo O, Schiavone N, Papucci L, et al: Down-regulation of nitric oxide synthase- 2 and cyclooxygenase- 2 pathways by p53 in squamous cell carcinoma. Am J Pathol 163: 723-732, 2003.

15. Kyzas PA, Stefanou D and Agnantis NJ: COX-2 expression correlates with VEGF-C and lymph node metastases in patients with head and neck squamous cell carcinoma. Mod Pathol 18: 153-160, 2005.

16. Alsner J, Sorensen SB and Overgaard J: TP53 mutation is related to poor prognosis after radiotherapy, but not surgery, in squamous cell carcinoma of the head and neck. Radiother Oncol 59: 179-185, 2001.

17. Mineta H, Borg A, Dictor M, Wahlberg P, Akervall J and Wennerberg J: p53 mutation, but not p53 overexpression, correlates with survival in head and neck squamous cell carcinoma. Br J Cancer 78: 1084-1090, 1998.

18. Nylander K, Dabelsteen E and Hall PA: The p53 molecule and its prognostic role in squamous cell carcinomas of the head and neck. J Oral Pathol Med 29: 413-425, 2000.

19. Dijkema IM, Struikmans H, Dullens HF, Kal HB, van der Tweel I and Battermann JJ: Influence of p53 and bcl-2 on proliferative activity and treatment outcome in head and neck cancer patients. Eur J Cancer B Oral Oncol 36: 54-60, 2000.

20. Hitt R, Ciruelos E, Amador ML, et al: Prognostic value of the epidermal growth factor receptor (EGRF) and p53 in advanced head and neck squamous cell carcinoma patients treated with induction chemotherapy. Eur J Cancer 41: 453-460, 2005.

21. Subbaramaiah K, Altorki N, Chung WJ, Mestre JR, Sampat A and Dannenberg AJ: Inhibition of cyclooxygenase-2 gene expression by p53. J Biol Chem 274: 10911-10915, 1999.

22. Erkinheimo TL, Lassus H, Finne P, et al: Elevated cyclooxygenase-2 expression is associated with altered expression of p53 and SMAD4, amplification of HER-2/neu, and poor outcome in serous ovarian carcinoma. Clin Cancer Res 10: 538-545, 2004. 
23. Lee DW, Park SW, Park SY, Heo DS, Kim KH and Sung MW: Effects of p53 or p 27 overexpression on cyclooxygenase- 2 gene expression in head and neck squamous cell carcinoma cell lines. Head Neck 26: 706-715, 2004.

24. Chen Z, Zhang X, Li M, et al: Simultaneously targeting epidermal growth factor receptor tyrosine kinase and cyclooxygenase-2, an efficient approach to inhibition of squamous cell carcinoma of the head and neck. Clin Cancer Res 10: 5930-5939, 2004.

25. Wirth LJ, Haddad RI, Lindeman NI, et al: Phase I study of gefitinib plus celecoxib in recurrent or metastatic squamous cell carcinoma of the head and neck. J Clin Oncol 23: 6976-6981, 2005.

26. Nishimura G, Yanoma S, Mizuno H, Kawakami K and Tsukuda M: A selective cyclooxygenase-2 inhibitor suppresses tumor growth in nude mouse xenografted with human head and neck squamous carcinoma cells. Jpn J Cancer Res 90: 1152-1162, 1999.

27. Sivula A, Buskens CJ, van Rees BP, et al: Prognostic role of cyclooxygenase-2 in neoadjuvant-treated patients with squamous cell carcinoma of the esophagus. Int J Cancer 116: 903-908, 2005 .
28. Ranelletti FO, Almadori G, Rocca B, et al: Prognostic significance of cyclooxygenase-2 in laryngeal squamous cell carcinoma. Int J Cancer 95: 343-349, 2001.

29. Victorzon M, Nordling S, Haglund C, Lundin J and Roberts PJ: Expression of p53 protein as a prognostic factor in patients with gastric cancer. Eur J Cancer 32A: 215-220, 1996.

30. Lim SC, Park SY and Do NY: Correlation of cyclooxygenase-2 pathway and VEGF expression in head and neck squamous cell carcinoma. Oncol Rep 10: 1073-1079, 2003.

31. Soo R, Putti T, Tao Q, et al: Overexpression of cyclooxygenase2 in nasopharyngeal carcinoma and association with epidermal growth factor receptor expression. Arch Otolaryngol Head Neck Surg 131: 147-152, 2005

32. Mrena J, Wiksten JP, Thiel A, et al: Cyclooxygenase-2 is an independent prognostic factor in gastric cancer and its expression is regulated by the messenger RNA stability factor HuR. Clin Cancer Res 11: 7362-7368, 2005.

33. Taylor D, Koch WM, Zahurak M, Shah K, Sidransky D and Westra WH: Immunohistochemical detection of p53 protein accumulation in head and neck cancer: correlation with p53 gene alterations. Hum Pathol 30: 1221-1225, 1999. 